\title{
V. Alphabetisches Register nach der Materie.
}

A.

Abstand von der Küste, unsichere Schätzung desselben

- s. a. Lufterscheinungen.

Aegir, norwegischer Dampfer, trifft am 10. März 1878 die am 8. dess. Mts. von der Mannschaft verlassene Galiote Yskea von Emden in der

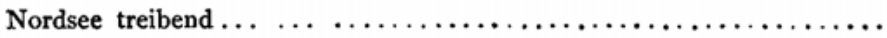

Aethelberth, britische Bark; Zusammenstoss derselben mit der britischen Brigg ${ }^{\text {Brotherly Love auf der Elbe am 14. Juli } 1878 \ldots \ldots \ldots \ldots}$

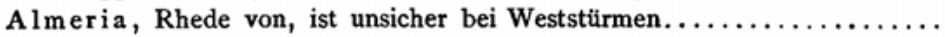

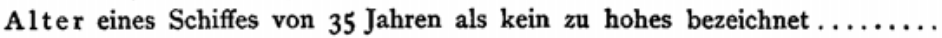
Alwine, Schaluppe von Wolgast; Zusammenstoss derselben mit der dänischen Jacht Elise am 8. Juli 1878 im Peenemünder Rack; Sinken derselben Amaranth, Bremer Schiff. Kapitän Brauer nimmt einen Theil der Mannschaft der Danziger Bark >Otto Georgee, welche im Boot ihr Schiff

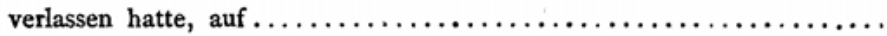

A mor, norwegische Bark; nimmt die Mannschaft der sinkenden deutschen

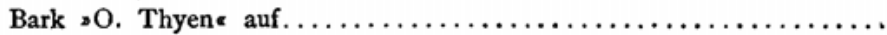

Anbohren des Schiffes seitens des Schiffers als muthmaassliche Ursache des

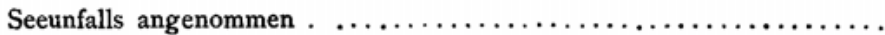

Anglo Norman, britische Bark; nimmt die Mannschaft der sinkenden deutschen Brigg 'Johann 4 auf, Seite 550, desgl. der Bark •Ada $4 .$.

Anker, Unklarwerden d̦esselben als Ursache eines Seeunfalls angenommen

- Reserveanker wird zweckmässiger beim Grossmast, als im Zwischendeck

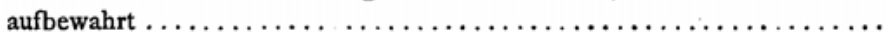

- s. a. u. Werfen.

Ankerketten, Nichtbefestigen derselben im Schiff, wodurch das Verankern eines Schiffes verhindert wurde, als ein Fehler bezeichnet..........

Ankerlaterne, Nichtbrennen einer solchen als Grund einer Kollision an-

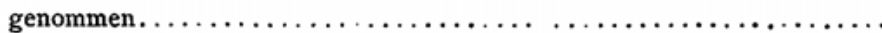

Anstecken eines mit Petroleum beladenen Schiffes beim Verlassen desselben durch die Mannschaft, im Interesse der Schiffahrt als gerechtfertigt an-

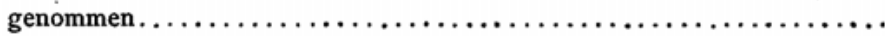

Ariel, Dampfer aus Nyborg; trifft am 27. Februar 1878 den Schooner sOtto \& Frieda\&, welcher, von der Mannschaft verlassen, angesichts

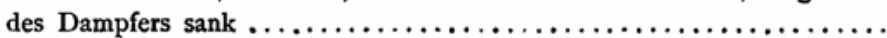


A ufgeben, zu frühzeitiges, eines Schiffes, ehe der Versuch gemacht, dasselbe mit den Pumpen flott zu erhalten, getadelt und als Mitursache eines Seeunfalls angenommen $\ldots \ldots \ldots \ldots \ldots \ldots \ldots \ldots \ldots \ldots \ldots$.

Aufstossen s. u. Wrackstück.

Ausguckmann; Unaufmerksamkeit desselben als Ursache eines Seeunfalls

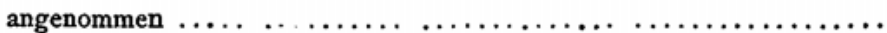

- Verwendung desselben zum Lothen als ordnungswidrig bezeichnet......

Auswässerung von $8-9$ Zoll bei $5 \frac{3}{4}$ Fuss Tiefgang als genügend

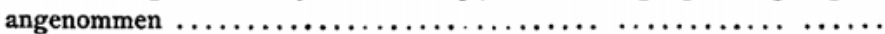

- desgl. von $3 / 4$ Fuss bei einem Fahrzeug von 4 Fuss Tiefgang ..........

- von wenigstens 2 Zoll auf den Fuss Tiefgang zur Seetüchtigkeit eines

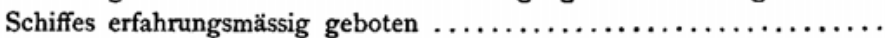

- von 15 Zoll bei $9^{1 / 4}$ Fuss Tiefgang für ausreichend gehalten ...........

- nach den Statuten und Versicherungsbedingungen der ostfriesischen Assekuranz-Gesellschaften nur eine Auswässerung von 1 Zoll auf den Fuss Tiefgang bei Fahrzeugen von $50-60$ Last verlangt .........

- s. a. u. Ueberladung.

\section{B.}

Ballast; Einnahme von solchem bei Holzladungen, falls keine Decklast genommen, wird als nicht gebräuchlich bezeichnet...............

- Nichteinnahme von solchem als ein Fehler gerügt, wenngleich dieselbe in Russland bei einer gewissen Art Fahrzeuge gebräuchlich ist .....668.669

Barre von Lagos; Passiren derselben während der Regenmonate als

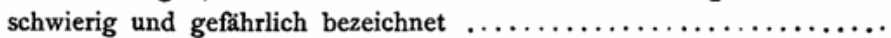

Beaufsichtigung, unterlassene, eines zu Anker liegenden Fahrzeugs seitens des Schiffers, als Pflichtwidrigkeit bezeichnet .............

Befugnisse, gewerbliche. Eine Theilung derselben nach der Verschiedenheit der Schiffe ist sowohl beim Schiffer- als Steuermannsgewerbe unzulässig

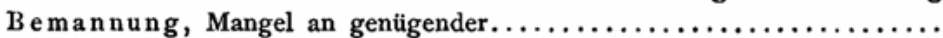

- Pflicht des Schiffers, für hinreichende $z$ sorgen $\ldots \ldots \ldots \ldots \ldots \ldots$

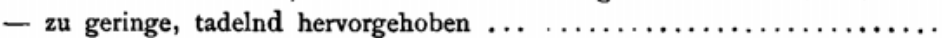

- von nur zwei Mann für seegehende Fahrzeuge als ungenügend bezeichnet.............................. r 59.162.246.249

- von zwei Mann bei seegehenden Fahrzeugen von 50 Tons Gehalt als ungenügend, aber üblich bezeichnet $\ldots \ldots \ldots \ldots \ldots \ldots \ldots \ldots \ldots . . . \ldots \ldots \ldots$

- von drei Mann als durchaus erforderlich für wirklich in See gehende

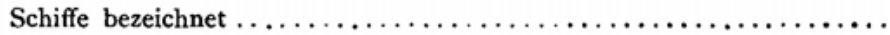

- Usance der ostfriesischen Küsten- und Wattfahrer nur zwei Mann Besatzung zu haben. Die Versicherungsgesellschaften verlangen ein Mehreres nicht

B e ra u bung eines an der chinesischen Küste gestrandeten deutschen Schiffes

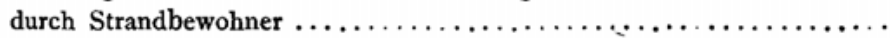

Besichtiger, amtliche. Die Anordnung von solchen betreffs der Reparatur eines Schiffes für nicht. ausreichend angenommen ...............

Beurkundung des Personenstandes s. Gesetzesstellen.

Bew achsensein des Schiff bodens als Ursache der schlechten Steuerfähigkeit eines Schiffes angenommen $\ldots \ldots \ldots \ldots \ldots \ldots \ldots \ldots \ldots \ldots . . . \ldots \ldots$ 
Bl ond e, deutscher Dampfer; nimmt die Mannschaft des sinkenden deutschen

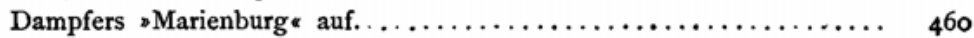

Böttcherholz zur Unterlage der Ladung verwendet, als genügender Ersatz

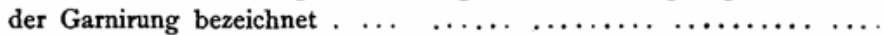

B oot; Ausrüstung eines grösseren Schiffes mit nur einem, tadelnd hervor-

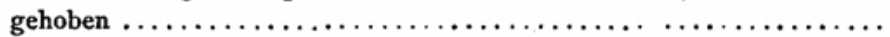

- Mitführen eines solchen seitens der kleineren Wattfahrzeuge nicht als unbedingt erforderlich erachtet und deshalb nicht als ein Mangel in der

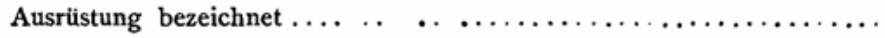

Brotherly Love, britische Brigg von Shields, in Folge Kollision mit der britischen Bark "Aethelberth $\propto$ am 14. Juli 1878 auf der Elbe gesunken

Bruch des Grossmastes in Folge von heftigem Schlingern ......... 204.206 Bundesk on sulate s. Gesetzesstellen.

Bunker; rechtzeitiger Verschluss der Schotten vor den Eingängen zu den

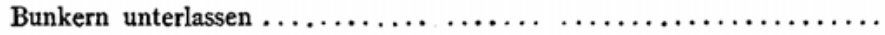

\section{C.}

Cays, Nuevo und Arena; Warnung der Segelanweisung, sich denselben bei Nacht zu nähern, da sie schlecht zu sehen und nicht anzulothen sind.

Charlie Blackwood, britischer dreimastiger Schooner; Kapitän Richardson. Trifft am 8. März 1879 die deutsche Galiote "Yskea and nimmt die Mannschaft derselben, welche ihr beschädigtes Fahrzeug verlassen

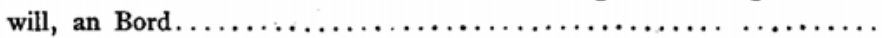

Chilian, Dampfer; versucht einen Theil der Mannschaft der Danziger Bark

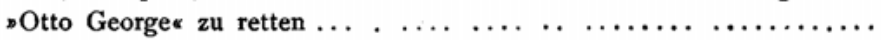

Christine, dänische Brigg; nimmt die Mannschaft des auf der Ladung

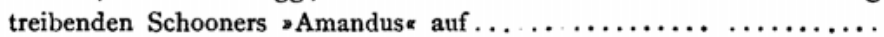

Chronometer; unterlassene Regelung eines solchen getadelt $\ldots \ldots \ldots \ldots$

Civilprozessordnung s. Gesetzesstellen.

Condor, schwedische Bark; nimmt die Mannschaft der sinkenden deutschen Brigg "Grossherzog Friedrich Franz" in der Nordsee auf..........

Consiglia Galatola, italienische Bark; nimmt die Mannschaft des sinken-

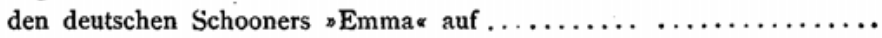

\section{D.}

Deck, Verlassen desselben seitens des Steuermanns während seiner Wache, und während das Schiff sich in gefährlicher Lage befindet, als Mangel gewöhnlicher Vorsicht und Pflichttreue bezeichnet ...............

Deckladung; eine solche von drei Lagen Sleepers als gebräuchlich bei

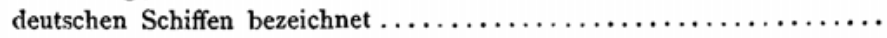

- s. a. Dynamit.

Decklast bei Holzladungen gebräuchlich und nicht verboten...........

- Der ungünstige Einfluss einer solchen auf die Seetüchtigkeit als erwiesen angenommen und deshalb ein gesetzliches Verbot als wünschenswerth bezeichnet. Die Stauung der Decklast in einer Höhe von $2^{1 / 2}$ Fuss bis einige Zoll unter der Regeling als die übliche Praxis in den Ostsee-

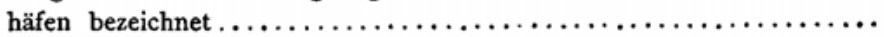


Deviation. Der Kompass im finnischen Meerbusen ist Unregelmässigkeiten

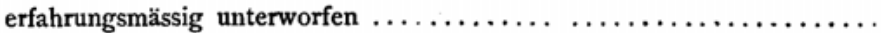

Domesnees, Riff von; als ein sehr gefährliches bezeichnet, da dort festgerathene Schiffe des Treibsandes wegen fast nie wieder loskommen

Durchbrechen eines auf Grund stehenden Fahrzeugs in Folge Unterwühlens des Grundes durch die Strömung als Ursache eines Seeunfalls

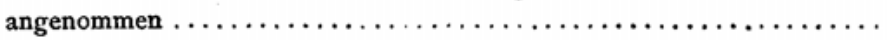

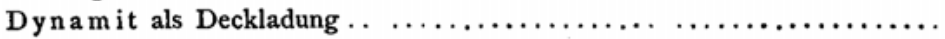

Seite

579

E.

East London am Cap der guten Hoffnung. Unsichere Rhede......... Ebbe und Fluth, Beobachtung derselben als Pflicht des Schiffers bezeichnet Ei der s. u. Feuerschiff; Lootsengaliote.

Entreprise, britische Smack; nimmt die Mannschaft des sinkenden deutschen Schooners $>$ Kronprinz Ernst Auguste auf...............

F.

Fahrgeschwindigkeit; Ermässigung derselben von 14 auf II Knoten als nicht hinreichend bei starkem Nebel bezeichnet, und entspricht nicht der Verordnung vom 23. Dezember 1871, Art. 16.

F anchon, Brigg von Rostock; leistet dem Stralsunder Schooner sCarolinea

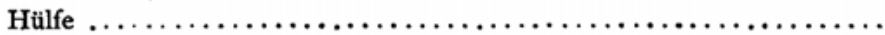

Feuer; Entstehung desselben durch die Reibung von mit Creosot getränkten Eisenbahnschwellen, als wahrscheinlich angenommen..........740.74I

- durch Blitzschlag entstanden, als Ursache eines Seeunfalls angenommen 663

Feuerschiff der Eider rettet die Mannschaft der sinkenden Tjalk s Heroe

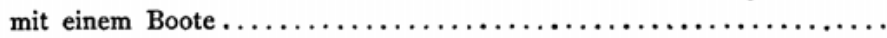

- von Falsterbo. Der Umstand, dass die beiden Feuer dieses Feuerschiffs in gleicher Höhe angebracht sind, sich also unter gewissen Umständen decken und wie ein Feuer erscheinen, als unzweckmässig bezeichnet..

- von Minsenersand als Marke zur Einsegelung in die Jade ............

Feuerschiffe; Einziehung der von der russischen Marine ressortirenden.

Fischerfeuer, Sichtweite derselben höchstens zwei Seemeilen..........

- s. a. Helder.

Flora, deutsches Schiff; macht einen Versuch die Mannschaft des gestrandeten Schiffes $\rightarrow$ Matterhorna zu retten. Vier Mann der Besatzung

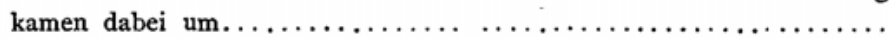

Fluthströmung, Richtung und Schnelligkeit derselben an der englischen

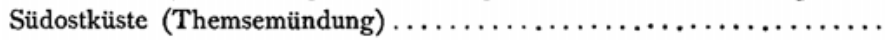

Frost, starker und plötzlich eintretender, wodurch Takelage und Ankergeschirr unregierbar wurden, als Hauptursache eines Seeunfalls an-

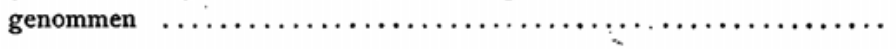

G.

Gaetano, italienische Bark, nimmt die Mannschaft der auf der Ladung

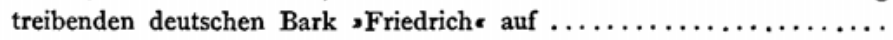
Garnirung, Mangel derselben bei einer Ladung Pflastersteine. 
Garnirung von Matten bei Salzladung als nicht genügend bezeichnet, sondern eine Garnirung wie bei trockener Ladung üblich, für erforderlich

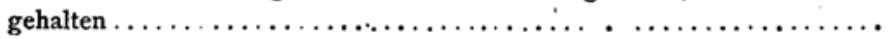

- Holz-; Mangel einer solchen bei einer Ladung Roheisen nicht als Mangel

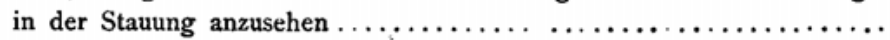

Gast on \& Lin a, französischer Schooner; rettet die Mannschaft der sinkenden deutschen Galeasse ${ }^{2}$ Sophie $\ldots \ldots \ldots \ldots \ldots \ldots \ldots \ldots \ldots .627 .828$

Geschäfts ordnung für das Ober-Seeamt s. Gesetzesstellen.

Geset $z$ vom 9. Mai 1853 s. Gesetzesstellen.

Geset $z$, Reichs-, betr. die Untersuchung von Seeunfallen s. Gesetzesstellen. Getreideladung s. u. Ladung; s. u. Schotten.

Glengarry, britischer Dampfer; nimmt einen Theil der Mannschaft und der Passagiere des sinkenden deutschen Dampfers sPommeraniac auf..

Gravelinors, französischer Schleppdampfer; nimmt die Mannschaft des gesunkenen deutschen Schooners sJohannac auf ..............

H.

Handelsgesetzbuch s. Gesetzesstellen.

Hannoversches Maass; Umrechnung desselben auf Grund der Maassund Gewichtsordnung vom 17. August 1868, Art. $21 \ldots \ldots \ldots \ldots \ldots$

Hartwell-Riff, wahrscheinliche Strandungsstelle der Schoonerbrigg

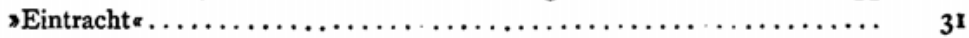

Helder, Leuchtfeuer; Verwechselung desselben mit Fischerfeuern ..... 513

Hermann, deutsche Galiote; nimmt die Mannschaft des im sinkenden $\mathrm{Zu}$ stande befindlichen Schooners Margarether auf................

Hülfe, Nichtleisten von, seitens der Schoonerbrigg $>$ W. Brügmann \& Sohn No I\&, Schiffer Schmidt, an die in Noth befindliche Bark s Mariac

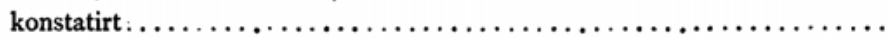

Hülfeleistung, Anerkennung der von dem britischen Dampfer ,Tealc nach der Kollision dem Lootsenschooner wWesere geleisteten ........

- des britischen Dampfers .Ferndalek an die deutsche Bark sEbenezar ..

- s. a. u. Strandvogt.

Jade, Verwechselung der Fahrwassertonnen beim Einsegeln in dieselbe... 41.42

- s. a. u. Lootsen.

John Ormst on, britischer Dampfer; nimmt die Mannschaft der deutschen Bark sMariac, welche im sinkendem Zustande verlassen war, auf..... 780

\section{$\mathbf{K}$.}

Kappen des Grossmastes, welcher gebrochen war, unterlassen, um die Manövrirfähigkeit nicht zu verringern und weil befürchtet wurde, derselbe würde beim Sturz den Fockmast mitreissen $\ldots \ldots \ldots \ldots \ldots \ldots \ldots$.

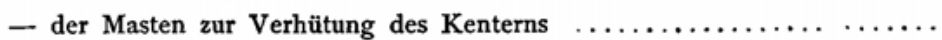

$\mathrm{K}$ arte der Gewässer an der argentinischen Küste, unrichtige Angabe betr., die Strömung enthaltend . . . . . . . . . .

- Special-; Mangel einer solchen als Mitursache eines Seeunfalls angenommen 568 
Karten; Unrichtigkeit derselben als Ursache eines Seeunfalls angenommen

- mangelhafte Ausrüstung mit solchen als Mitursache eines Seeunfalls

bezeichnet................................ 153.576

- s. a. Segelanweisung.

Kentern eines Schiffsbootes Seite $\mathbf{1 6 2}$; desgl. bei dem Versuch, die Insel

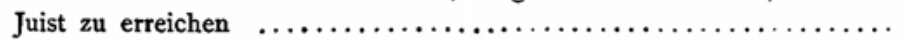

- eines mit Tonnenplanken beladenen Schooners..................

- s. a. u. Kappen, Kettestecken.

Kentish Knock, Bank; Strandung der Hamburger Bark »Auguste Solchere

- Leuchtfeuer; Verwechselung desselben mit Sunk-Feuerschiff bezw. mit einem Fischerboot als Ursache der Strandung der Brigg „Eos๔ angenommen

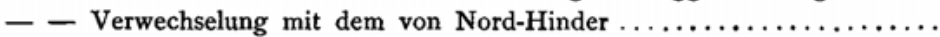

Kettestecken im Zusammentreffen mit einem Windstosse als Ursache des Kenterns eines eben vom Stapel gelassenen Schiffes angenommen ....

Klassifizirung eines Schiffes kann als Beweis für die Seetüchtigkeit eines solchen nicht unbedingt gelten, wenn die Klassifizirung vor längerer

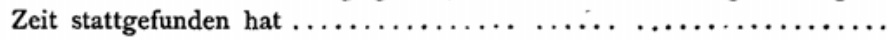

Klinker. Gewicht eines Klinkersteins auf reichlich $4 \frac{1}{2}$ Pfund angenommen

Küstenströmung; Versetzung durch dieselbe als Grund der Strandung

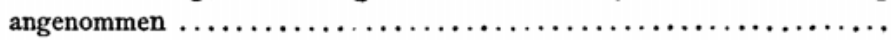

Kung-Kung-Tan, Inseln bei Chefoo (China); Strandungsort der Bark -Cerese von Hamburg

Seite

240

150

$55^{8}$

4

97

4

$8 \mathbf{r}$

420

68

I.

Ladepforten; Eindrücken derselben durch Eis auf der Elbe als Ursache

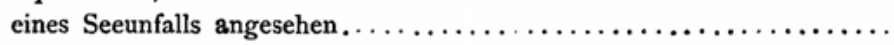

Laderaum, Zutritt in denselben von der Proviantkammer seitens der Mannschaft wird als ordnungswidrig bezeichnet...............

Ladung; Gewicht derselben beträgt gewöhnlich 10-40 Prozent mehr als die Vermessung des Schiffes ergiebt $\ldots \ldots \ldots \ldots \ldots \ldots \ldots \ldots \ldots \ldots \ldots \ldots$.

- Getreide-; Sacken (Zusammenfallen) einer solchen als Ursache des Ueber-

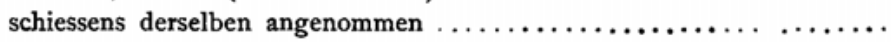

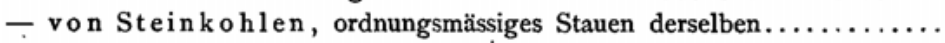

- - zählt nicht zu den gefährlichen Ladungen, weil sie über den ganzen

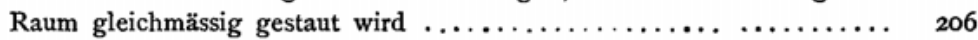

- Stein-, nassgewordene, als Ursache des Verlustes der Tjalk „Foskea '

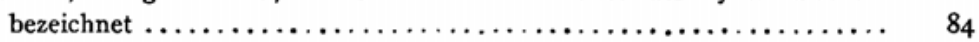

- Schiffe mit, leck geworden und gesunken .................65.67

— zu schwere, als Ursache des Leckwerdens angenommen ........... 69

- s. a. u. Böttcherholz, Schiffsjournal, Schwergut, Selbstentzündung, Ueberladung.

Ladungsfähigkeit eines Schiffes übersteigt die durch die Vermessung

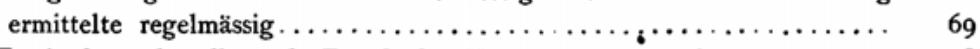

— Ermittelung derselben als Zweck der Vermessung anzusehen $\ldots \ldots \ldots$ i18

- die durch die Vermessung ermittelte als nicht maassgebend bezeichnet 275

- s. a. u. Schiffsvermessung. 
Längsschotten s. Schotten.

Seite

L andrecht s. Gesetzesstellen.

Leck; der Versuch einen solchen durch Unterziehen eines Segels zu verstopfen,

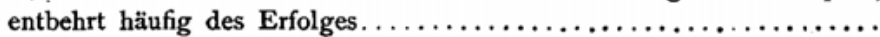

- s. a. u. Seetüchtigkeit.

Leckspringen des Schooners sMargarethas in Folge schlechten Wetters

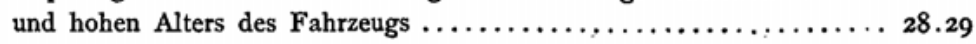

- s. a. u. Schwefelsäure; s. a. u. Seitenwände.

L eckwasser s. u. Salpeter.

Leuchtfeuer-Verzeichniss; der Besitz eines solchen von neuestem Datum wird als Pflicht eines Schiffers bezeichnet................

Liffey, britische Fregatte; leistet der am 18 . Juli 1878 in der Nähe der kanarischen Inseln in Brand gerathenen Hamburger Bark "Anita " Hülfe und bringt die gerettete Mannschaft derselben nach St. Vincent

Loggen und Lothen. Vernachlässigung dieser gewöhnlichsten Vorsichtsmaassregeln als Verschulden an einem Seeunfall angenommen ........

Lootsen. Auslegung des Gesetzes, betr. die Untersuchung von Seeunfällen, in Bezug auf Beurtheilung der Handlungen oder Unterlassungen der

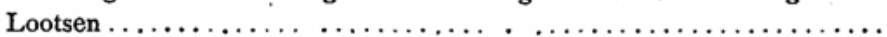

- Die hamburgischen Elblootsen sind nach gerichtlicher Entscheidung nicht als Zwangslootsen im Sinne des Artikels 780 des Handelsgesetzbuches anzusehen

- brauchen auf der Elbe nicht genommen zu werden ...............

- Falsche Auffassung eines solchen betreffs der Verpflichtung eines Segelschiffes zum Warten bis ein Dampfer passirt ist, wenn dieser ein Signal

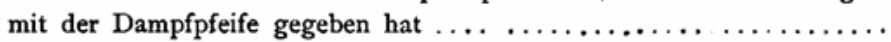

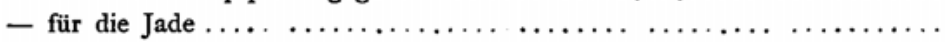

- Handlungen und Unterlassungen derselben bei Führung von Schiffen . 52.53

- Nichtbefolgung der Anweisungen eines solchen. Verpflichtung des Schiffers zur Befolgung der Anweisung eines Zwangslootsen ........... 35.35.39

- Nichteinnahme eines Standorts, der einen klaren Ueberblick gestattete, wird einem Lootsen als Verschulden ausgelegt .................

- Pflicht derselben, nach einem Seeunfall, bei welchem sie die Führung eines Schiffes gehabt, sich von den Umständen und Ursachen genauere Ueberzeugung zu verschaffen Seite 194; desgl. sich von der Richtigkeit der Kompasse auf den ihrer Führung anvertrauten Schiffen zu überzeugen

- Unfähigkeit oder Nachlässigkeit eines solchen als Ursache eines Seeunfalls

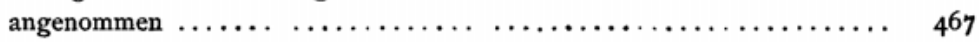

- Verschulden eines solchen an einem Seeunfall angenommen $\ldots \ldots \ldots \ldots$ 138

Lootsenfahrzeug s. u. Toplaternen.

Lootsengaliote in der Eider. Das Rettungsboot derselben leistet dem gestrandeten Ever sElisabeth « Hülfe................... Ior

Lootsenkutter, Verwechselung mit einem Fischerfahrzeug .......... 75

L oots enordnung s. Gesetzesstellen.

Lootsenzwang besteht auf der Weser nicht $\ldots \ldots \ldots \ldots \ldots \ldots \ldots \ldots, 681$

Loth, mangelhafte und unaufmerksame Benutzung desselben $\ldots \ldots \ldots \ldots 3.4: 5$

- ungenügender Gebrauch desselben als Strandungsursache bezeichnet .... 210

- Nichtgebrauch desselben bei unsicherem Besteck in der Nordsee wird dem Schiffer als Verschulden angerechnet $\ldots \ldots \ldots \ldots \ldots \ldots \ldots \ldots \ldots \ldots, 508$ 


\section{XXVI}

Lothen, Unterlassung desselben als Schuld des Schiffers bezeichnet .......

- desgl. als Ursache eines Seeunfalls angenommen..$\ldots \ldots \ldots \ldots \ldots \ldots$.

- Vornahme desselben als besonders erforderlich bei der Schiffahrt über den Watten bezeichnet.............................. 113

Lothungen, Nichtvornahme derselben wird entschuldigt .......... 288

- Zwecklosigkeit derselben auf der Campechebank ............ $27 \mathbf{I}$

L ufterscheinungen als Ursache der Ueberschätzung des A Abstandes vom

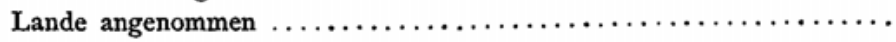

III.

Maass - und Gewichts ordnung s. Gesetzesstellen.

Maria, Schooner von Marstall, nimmt die Mannschaft des gesunkenen Schooners \&Otto und Frieda von Rostock auf und landet dieselbe bei

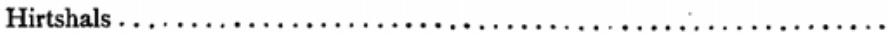

Marie, norwegische Bark; Schiffskapitän Botner. Nimmt die Mannschaft des deutschen Schooners $₫$ Zwei Gebrüderc, welche ihr sinkendes Fahr-

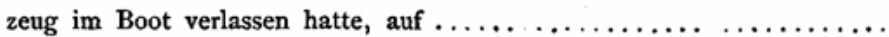

Mellneraggen bei Memel, Strandungsort der Bark »Adelheid « aus Kiel Mellum-Plate als Strandungsort der Tjalk sFoskea angenommen. . . Merchant shipping act s. Gesetzesstellen.

Minsenersand s. u. Feuerschiff.

Möwenhaken, Einengung des Swinefahrwassers durch denselben ......

N.

Nebel als Ursache der Strandung des Schooners sAdelheid» bezeichnet

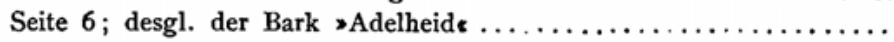

- als wahrscheinliche Ursache der Strandung des britischen Dampfers

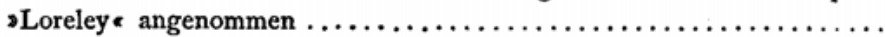

- richtige Anwendung von Vorsichtsmaassregeln bei Nebel ...........

- s. a. Fahrgeschwindigkeit.

Nebelhörner, Nichtvorhandensein ihren Zwecken entsprechender.....

Nebelhorn, ununterbrochener Gebrauch desselben ..................

Nebelhornstation; Einrichtung einer solchen bei Moruptange wird als

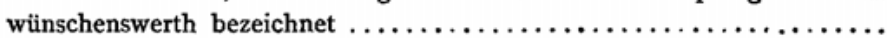

Nebelsignale; abweichende Angaben über das Hören derselben. Einwirkung der Schallrichtung und des Geräusches der Maschinen......62.63

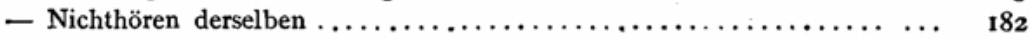

Nichtbeachtung der Befehle des Kapitäns seitens des Steuermanns als Verschulden an einen Seeunfall angenommen $\ldots \ldots \ldots \ldots \ldots \ldots \ldots, \quad 76$

Nord-Hinder, Feuerschiff, Verwechselung mit dem von Kentish-Knock . 4

Nothflagge, erfolgloses Zeigen derselben .................... 68

Nothsigna 1, Mangel des vorschriftsmässigen $\ldots \ldots \ldots \ldots \ldots \ldots \ldots \ldots$, 108

Nothsignale, Nichtgebrauch derselben wird dem Schiffer zur Last gelegt 283

- Unkenntniss derselben wird dem Schiffer und Steuermann zum Vorwurf

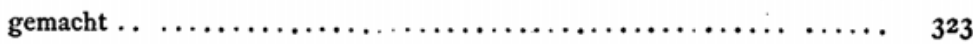

Noth-und Lootsen-Signal-Ordnung s. Gesetzesstellen. 


\section{P.}

Pampero, südöstlicher Sturm; als Ursache der Strandung eines Schiffes

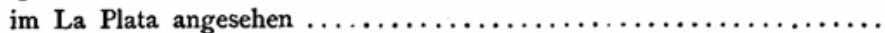

Peilungen, Vornahme von solchen als eine gebotene Vorsicht bei der Navigirung in engen und gefährlichen Fahrwassern bezeichnet ....... Pendle-Hill, britisches Schiff von Whitehaven, nimmt die Mannschaft der sinkenden Brigg ${ }^{2}$ Superbs von Hamburg auf...............

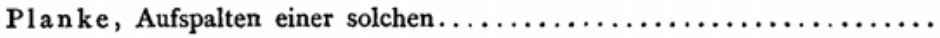
Polizei-Ordnung s. Gesetzesstellen.

Port Elizabeth; Mangel daselbst an Einrichtungen zur gründlichen

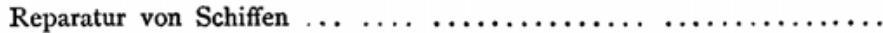

Pumpen, unklare, als Grund zum Aufgeben des Schiffes angesehen .....

- Unklarwerden derselben bei einer Ladung Düngersalz als Ursache eines

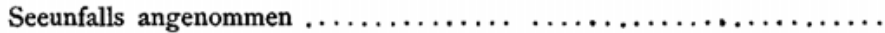

- Verstopfen derselben bei einer Ladung Thonerde.. ..............

- desgl. durch Ladungstheile (Palmkerne), als Ursache eines Seeunfalls an-

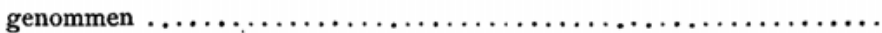

- s. a. u. Schwungräder.

Pumpenraum, Abschluss desselben gegen das Eindringen von Sandballast

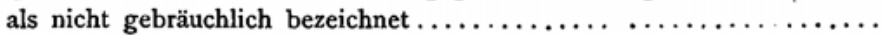

Pym, britisches Schiff, Kapitän Stapleton; rettet die Mannschaft der Schoonerbrigg Berthak am 19. Mai 1878 im südatlantischen Ozean

Seite

Querschotten s. u. Schotten.

\section{R.}

Raketen-A pparat; Anwendung desselben bei der Strandung der britischen Brigg $\gg$ Argyrac bei der Insel Juist $\ldots \ldots \ldots \ldots \ldots \ldots \ldots \ldots \ldots \ldots$

- Rettung der Mannschaft der Schoonerbark „Vertrauen« durch einen solchen an der Küste von Jütland $\ldots \ldots \ldots \ldots \ldots \ldots \ldots \ldots \ldots \ldots \ldots$

Regulations s. Gesetzesstellen.

Reichs-Oberhandelsgericht s. Gesetzesstellen.

Reikiavik, Hafen von; als unsicher bei stürmischem Wetter bezeichnet. . Reserveanker s. u. Anker.

Ret tungsboot, Helgoländer; rettet die Besatzung der Tjalk sCatharinae

- verspätetes Eintreffen des von Norderney bei einem Strandungsfall ....

- von Horumersiel; Versuch desselben zur Rettung der Mannschaft der

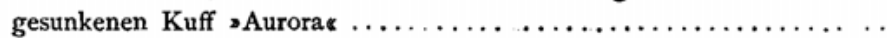

- - Juist; vergebliche Bemühung desselben, die gestrandete britische Brigg

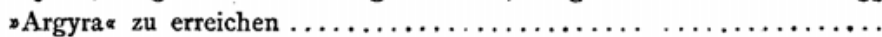

- Maassluis; rettet die Mannschaft der deutschen Schoonergaliote •Wodan

Rettungsbojen werden als zweckmässiger und nothwendiger Ausrüstungsgegenstand der Seeschiffe bezeichnet.$\ldots \ldots \ldots \ldots \ldots \ldots \ldots \ldots \ldots \ldots \ldots \ldots \ldots \ldots$

Rettungsgesellschaft, die nord- und südholländische. Ein Boot derselben rettet die Mannschaft des gestrandeten Schooners $>$ Maria....

Revision s. u. Seetüchtigkeit. 


\section{XXVIII}

Ruder; Unbrauchbarwerden desselben in Folge von Stossen des Schiffes

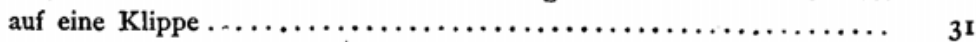

- Wirkung desselben bei rückwärtsgehender Schraube............. 65

Ruderkette, Bruch derselben.......................... 302

Ruderpfosten, Bruch des ............................. I

Sacken s. u. Ladung.

S.

Salpeter; Wegschmelzen einer Ladung durch Leckwasser ............

Scharfe (Quernaht); Springen einer solchen als unberechenbarer Zufall bezeichnet und als Ursache eines Seeunfalls angenommen.........

Schiffahrt; Mangel an für dieselbe bestimmten Hülfseinrichtungen als

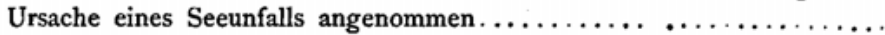

Schiffer; Mangel an Muth und Energie eines solchen als Ursache eines

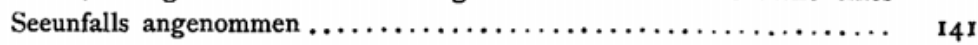

- Verantwortlichkeit desselben für die Navigirung seines Schiffes .... . 129

- s. a. u. Vereidigung, Verklarung, Verpflichtung.

$\mathrm{Schiffsboden} \mathrm{s.} \mathrm{Bewachsensein} \mathrm{desselben.}$

Schiffsjournal als wichtiges, zuweilen unersetzliches Beweismittel bezeichnet

- Erhaltung desselben nach einem Seeunfall dem Schiffer zur Pflicht gemacht

- Handelsgesetzbuch schreibt nicht vor, dass Anbringung von Schotten zur Sicherung der Ladung im Journal bemerkt werden muss ...........

- Nichtaufbewahrung desselben als ordnungswidrig getadelt...........

- Nichtmitnahme desselben beim Verlassen eines sinkenden Schiffes dem Schiffer zum Vorwurf gemacht $\ldots \ldots \ldots \ldots \ldots \ldots \ldots \ldots \ldots \ldots \ldots$

- Nichtunterschreiben desselben nach einem Seeunfall als ordnungswidrig

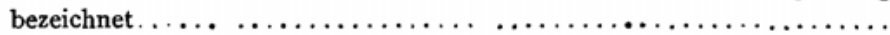

- Pflicht des Schiffers, die Führung desselben zu überwachen .........

- Unterlassene Rettung desselben dem Schiffer als eine tadelnswerthe Nach-

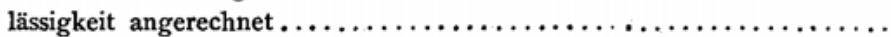

- s. a. u. Strandvogt.

$\mathrm{Schiffsrath;} \mathrm{Abhaltung} \mathrm{eines} \mathrm{solchen} \mathrm{ist} \mathrm{gesetzlich} \mathrm{nicht} \mathrm{geboten......}$

- Beschluss eines solchen entlastet den Schiffer nicht .................

Schiffsregister; Löschung eines verschollenen Schiffes in demselben.

Schiffsschraube; Wirkung einer linksschlagenden auf den Curs eines

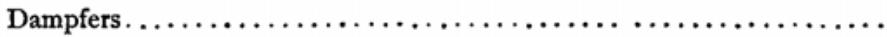

Schiffsvermessung. Mecklenburgische Verordnung vom 20. März 1867 bezeichnet als Zweck der Vermessung die Ermittelung der Höhe der $\mathbf{z u}$

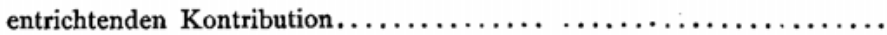

- Zweck derselben ist die Ladungsfähigkeit des Schiffes festzustellen, jedoch nicht eine unüberschreitbare Grenze für das Gewicht der Ladung

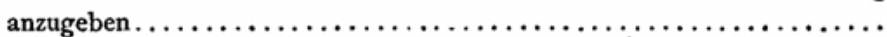

- s. a. u. Ladung, Tragfähigkeit.

Schiffsvermessungs-Ordnung s. Gesetzesstellen.

Schiffswände; Absetzen derselben mit aufrecht stehenden Eisenplatten bei einer Ladung Spiegeleisen, als anerkannt gute Maassregel bezeichnet

Schleppdampfer. Anwendung des §. I5 der Kaiserlichen Verordnung vom 23. Dezember 1871 auf dieselben 
Schlingern s. u. Bruch.

Seite

Sch otten. Empfohlen, den Verschluss der Schotten in den Kohlenbunkern so einzurichten, dass derselbe von Deck aus bewirkt werden kann ....

- Geweling-, Anwendung von solchen bei Küstenfahrern in der Ostsee

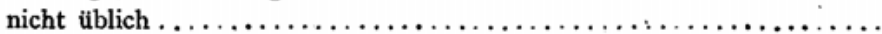

- Längs-, zur sicheren Stauung von Getreideladungen bei grösseren Schiffen nothwendig, bei kleineren jedoch nicht erforderlich, sondern besser durch Querschotten zu ersetzen $\ldots \ldots \ldots \ldots \ldots \ldots \ldots \ldots \ldots \ldots \ldots \ldots \ldots \ldots \ldots \ldots \ldots$.

Schotten; mangelhafte Einrichtung derselben als Mitursache eines Seeunfalls

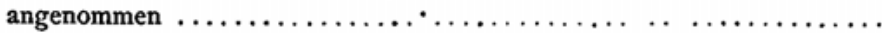

- s. a. u. Bunker, Schiffsjournal.

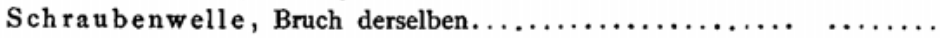

Schwefelsäure; schädliche Einwirkung derselben auf die eiserne Verbolzung eines Schiffes, als wahrscheinliche Ursache des Leckspringens

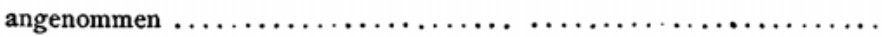

Schwergut. Verladung von Schwergut unten im Schiff und dadurch herbeigeführte heftige Bewegungen desselben.................

Schwimmgürtel; unrichtiger Gebrauch derselben seitens der Passagiere. Empfehlung, in Zukunft Abbildung dieser Apparate auf den Passagier-

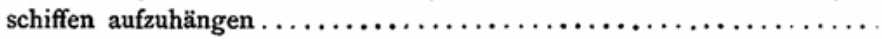

Schwungräder an den Pumpen als praktisch und empfehlenswerth

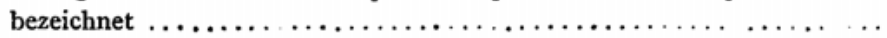

Se e a mt, Ober-. s. Gesetzesstellen.

Seemannsordnung s. Gesetzesstellen.

Seesch iffer- und Seesteuermanns-Prüfungen s. Gesetzesstellen.

Seetüchtigkeit; Bedeutung eines Leckes für dieselbe ........ 209.21r

- In kurzen Zwischenräumen vorzunehmende Revision bei alten Fahrzeugen ohne Metallhaut erforderlich bezeichnet, um die Seetüchtigkeit der-

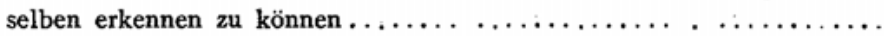

- s. a. n. Auswässerung.

See un fall-Gesetz s. Gesetzesstellen.

Seewarte, deutsche, Agentur in Emden; Witterungsbericht vom 8. Juni I 878 Seite 68 ; desgl. vom 2. August $1878 \ldots \ldots \ldots \ldots \ldots \ldots \ldots$

Segelanweisung von Jülfs \& Balleer (die Seehäfen etc. der Erde, Suppl. Band) betr. die Einfahrt nach Aux Cays. Unzuverlässigkeit

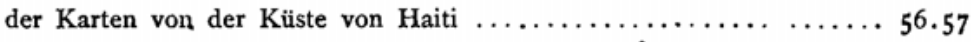

Segelkarte des südlichen Theiles der Ostsee zu'Preussens Seeatlas. Fehlerhaftigkeit derselben ist festgestellt betr. die Ostküste von Oeland. Lage der Susanna-Bank ........................ . 652.653

Seitenwände. Die Stelle zwischen Wind und Wasser als die bezeichnet, -welche am sorgfältigsten revidirt werden müsste, als dem Leckwerden

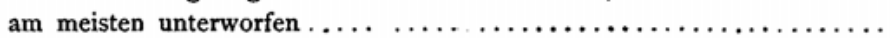

Seitenlichter; Nichtanzünden derselben nach Sonnenuntergang als eine Pflichtwidrigkeit des Schiffers bezeichnet $\ldots \ldots \ldots \ldots \ldots \ldots \ldots \ldots \ldots, 683$

- Nichtführen derselben als Ursache eines Seeunfalls angenommen .... 722.724

Selbstentzündung des zum Verstauen einer Ladung verwendeten Strohs als wahrscheinliche Entstehungsursache des Feuers in einem Schiffe

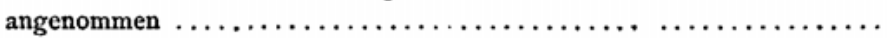


Selbstentzündung einer Hanfladung, welche mit Oel in Berührung gekommen, als Ursache eines Seeunfalls angenommen .............

- von feuchtem Stroh von dem Direktor des chemischen Staats-Laboratoriums in Hamburg als wahrscheinliche Ursache des Brandes an Bord

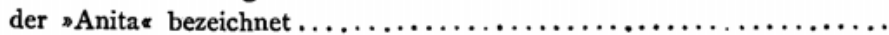

Sorgleinen; in dem Fehlen derselben ist ein Vorwurf gegen den Schiffer

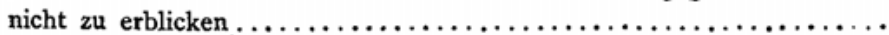

- Fehlen derselben nicht als mangelhafte Ausrïstung eines Schiffes angesehen Steinkohlen s. u. Ladung.

Steinladung s. u. Ladung.

St teuerf äh ig keit , mangelhafte, als muthmaassliche Ursache eines Zusammen-

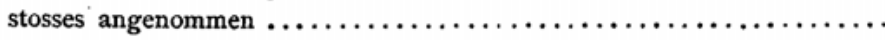

Seite

- s. a. Bewachsensein des Schiffsbodens.

Steuerkette; Ablaufen derselben von der Walze. Die in Folge desselben eingetretene Steuerlosigkeit als Ursache eines Seeunfalls angenommen..

Steuermann, Unterlassungen eines solchen als Verschuldung bei einem Seeunfall angenommen.$\ldots \ldots \ldots \ldots \ldots \ldots \ldots \ldots \ldots \ldots \ldots \ldots \ldots \ldots \ldots$.

- s. a. u. Vereidigung, Verklarung, Verpflichtung.

Steuermannsgewerbe, Mangel an den zur Ausübung desselben erforder-

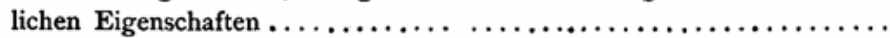

Strafprozessordnung s. Gesetzesstellen.

Strandung sordnung s. Gesetzesstellen.

Strandvogt. Ablehnung von Hülfeleistung durch einen solchen wegen

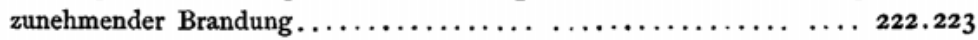

- Verpflichtung desselben zur Ansichnahme des Schiffsjournals und zum Abschluss desselben bei Strandungsfällen.................. 216

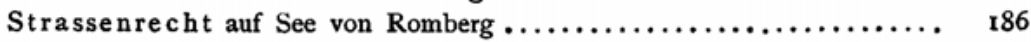

Strömung, Annahme einer südwestlichen an der Südküste von Haiti in den Monaten Januar bis März.........................

- Eine westlich setzende als Ursache der Strandung des Schooners $>$ Rugia angenommen. Regelmässiges Vorkommen derartiger Strömungen im östlichen Theil der Ostsee bei West- und SW-Winden $\ldots \ldots \ldots \ldots \ldots .24$

- Nichtbeachtung derselben als Hauptursache der Strandung bezeichnet ..

- Richtung derselben an der argentinischen Küste. Unkenntniss dieser Strömung als Ursache der Strandung des Dampfers »Karnake ange-

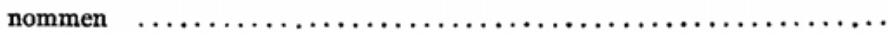

- Ungenügende Berücksichtigung der Südwestströmung bei Bonavista als Ursache der Strandung der Schoonerbrigg Eintracht $\propto$ angenommen 30.31 .32

- ungewöhnliche bei Haiti als Ursache der Strandung der Schoonerbrigg -Rebeccar angenommen .......................... 104.105

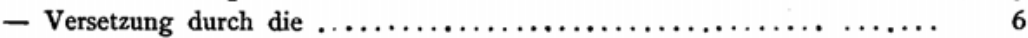

- Vorhandensein einer starken nach SW in der Nähe der Insel Bonavista (Kap Verdische Inseln). . . . . . . . . . . . . . . . . . . . .

- Vorherrschen, einer starken südlichen in der Nähe der jütischen Küste bei starken nördlichen Winden. Eine solche als Ursache eines Seeunfalls

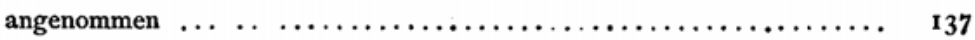

- vorherrschende, existirt nicht auf der Campechebank ............ 266 
Strömung s. a. u. Durchbrechen.

Seite

Strömungen, starke nach Norden setzende, herrschen erfahrungsmässig bei

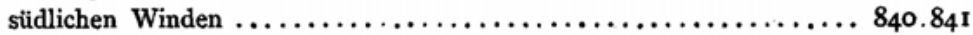

Sturzsee; schwere Beschädigung eines Schiffes durch eine solche $\ldots \ldots \ldots$ I47

T.

Teal, britischer Dampfer; Kollision desselben mit dem Lootsenschooner

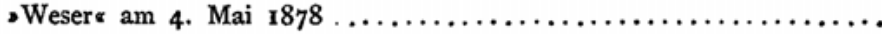

Tonnen s. u. Jade.

Toplaternen als karakteristisches Kennzeichen eines Lootsenfahrzeugs

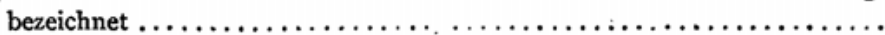

Tragfähigkeit eines Schiffes um ein Drittheil höher angenommen, als die Vermessung des Schiffes ergiebt $\ldots \ldots \ldots \ldots \ldots \ldots \ldots \ldots \ldots . . . \ldots \ldots$

Tragfähigkeit eines $\mathrm{Sch}$ iffes ist nicht ausschliesslich von seinem Raumgehalt, sondern auch von der Bauart und dem Material etc.

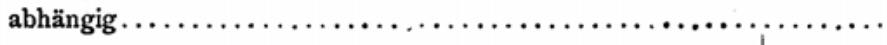

U.

Ueberladung eines deutschen Schiffes in Havre durch einen Agenten des Büreau Veritas und einen Kommissar der dortigen Kaufmannschaft

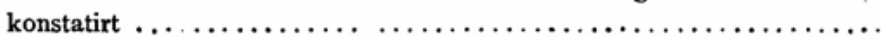

- eines Schiffes ist anzunehmen, wenn die Auswässerung desselben im Missverhältniss zum Tiefgang steht $\ldots \ldots \ldots \ldots \ldots \ldots \ldots \ldots \ldots \ldots, 28$

Ueberschiessen einer Ladung China-Clay .................. 7

Uebersegeln s. u. Wrack.

Umschlagen eines mit voller Takelage vom Stapel gelassenen Schiffes..

Verbolzung s. u. Schwefelsäure.

V.

Vereidigung von Schiffer und Steuermann zu unterlassen, wenn die Glaubwürdigkeit dieser Personen Zweifel unterliegt, sonst aber vorzunehmen.

Verklarung. Keine wesentliche Voraussetzung eines seeamtlichen Spruches

- Pflicht der Schiffsleute, besonders des Schiffers und Steuermanns, auf eine

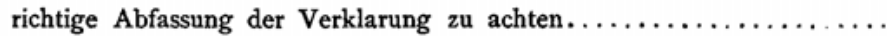

Vermes sung s. Schiffsvermessung.

Victoria, schwedische Bark; nimmt die Mannschaft des gesunkenen

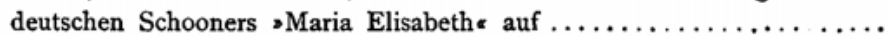

Ville de Fuveau, französische Bark; nimmt die Mannschaft des sinkenden deutschen Schiffes Andreas Rickmers $\&$ auf ..................

Verordnungen s. Gesetzesstellen.

Verpflichtung des Steuermanns, seinen Rath dem Schiffer auf Befragen, nach sorgfältiger Prüfung aller Umstände, zu ertheilen .............

Verschollenheit eines Schiffes konstatirt .................. 196

\section{W.}

Werfen von Bugankern und Ketten als unbesonnene Handlungsweise

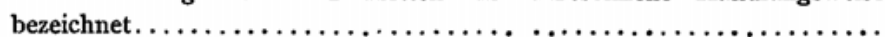

Wirbelsturm als Ursache des Seeunfalls der Bark sOtto Georgee angeführt 


\section{XXXII}

Seite

Wit tsand, Strandungsort des britischen Schooners "David...$\ldots \ldots \ldots .40$

Wrack, Uebersegeln eines treibenden, als Ursache eines Seeunfalls an-

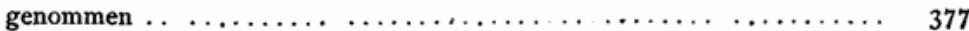

Wrackstü ck; Aufstossen auf ein solches als muthmassliche Ursache eines

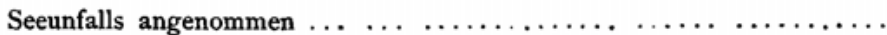

160

Z.

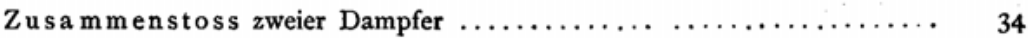

- eines Dampfers mit einem Fischerkutter $\ldots \ldots \ldots \ldots \ldots \ldots \ldots \ldots \ldots$ r $8 \mathbf{r}$

- eines Dampfers mit einem Leichterfahrzeug $\ldots \ldots \ldots \ldots \ldots \ldots \ldots \ldots$ 23I

- eines Dampfers mit einem Lootsenschooner . ................ 84

- eines Dampfers mit einem Segelschiff ........... 13.48.60.183.191.250

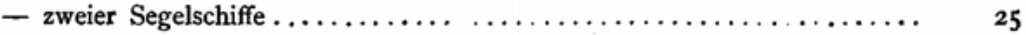

- s. a. u. Steuerfähigkeit.

Zusammenstossen von Schiffen auf See, Verordnung betr. Verhütung d. Z. s. Gesetzesstellen. 

